\title{
Los primeros elementos de hierro en Iberia Earliest iron objects in Iberia
}

\author{
Jesús R. Álvarez Sanchís ${ }^{1}$ \\ Alberto J. Lorrio Alvarado ${ }^{2}$ \\ Gonzalo Ruiz Zapatero ${ }^{3}$
}

\begin{abstract}
Resumen
La introducción de la metalurgia de hierro en la Península lbérica fue un proceso complejo en el que se pueden diferenciar dos vías de entrada. La primera y más importante fue la Mediterránea, que se inició en la etapa Pre-colonial y se intensifico durante la posterior colonización fenicia en el mediodía peninsular. Y la segunda, peor documentada, la continental desde el Sur de Francia a través de los pasos del Pirineo en la transición Bronce Final/ Primera Edad del Hierro. Se bosqueja luego la expansión del hierro hacia la Meseta y la región cantábrica. Se destaca que además de la pura dimensión tecnológica es importante considerar los factores económicos y sociales de las sociedades que incorporan el nuevo metal porque su comprensión es crucial para entender el proceso de adopción de la nueva tecnología. Se destacan las limitaciones actuales en torno al comienzo y adopción del hierro y finalmente se hace un ruego por conseguir datos más y mejor contextualizados en relación con el trabajo del hierro.
\end{abstract}

Palabras clave: Metalurgia del hierro, Península Ibérica, Bronce Final, Edad del Hierro, colonización fenicia, innovación tecnológica.

\begin{abstract}
The introduction of iron metallurgy in the Iberian Peninsula was a complex process in which it is possible to distinguish two ways of arrival. First, and the most important, the Mediterranean route was active since the pre-colonial period and intensified during the following stage of Phoenician colonization in the South of Iberia. And second, the continental way, extending this new technology from the south of France through the Pyrenees passes to the interior of Catalonia during the Late Bronze/Early Iron Age, although with poor data. The expansion to the center of Iberia and the North regions is outlined. Beside the strictly technological aspects it is important to also consider the social and economic factors of the communities that incorporate the new metal, and whose understanding is crucial to explain the process of adoption of the new technology. A consideration of contemporary limitations is stressed and finally, we make a plea for more contextualized data surrounding the iron working process.
\end{abstract}

Keywords: Iron metallurgy, Iberian Peninsula, Late Bronze Age, Iron Age, Phoenician colonization, innovation technology.

\section{INTRODUCCIÓN}

Aunque muchas veces invención e innovación se emplean como sinónimos desde la perspectiva de la historia tecnológica son conceptos claramente diferen- tes (Killick, 2015: 314)4. Una invención es la aparición inicial de una idea o proceso, tanto si es una verdaderamente nueva o se trata de una modificación distinta de una idea o proceso existente, mientras que una innovación es una invención que es ampliamente aceptada
1 Departamento de Prehistoria. Facultad de Geografía e Historia. Universidad Complutense de Madrid jralvare@ghis.ucm.es

2 Departamento de Prehistoria, Arqueología e Historia Antigua. Facultad de Filosofía y Letras. Universidad de Alicante alberto.lorrio@ua.es

3 Departamento de Prehistoria. Facultad de Geografía e Historia. Universidad Complutense de Madrid gonzalor@ghis.ucm.es
4 Este trabajo se ha realizado dentro del marco de los proyectos del Ministerio de Economía y Competitividad HAR2013-41447-P “E1 Bronce Final y la Edad del Hierro en el Sureste y el Levante de la Península Ibérica: procesos hacia la urbanización” y HAR201565994-R «Vettones: estudios de urbanismo y sociedad mediante técnicas no destructivas». 
(Renfrew, 1978: 89). En otras palabras "todas las innovaciones implican primero y por encima de todo una elección humana, que determina si la innovación será aceptada o rechazada a favor de la vieja práctica o material" (Renfrew, 1984: 405).

En las tecnologías modernas complejas esa transición de la invención a la innovación requiere generalmente "habilidades organizativas" para ensamblar los componentes- talento, finanzas, manufactura y marketing-y crear lo que Hughes (1989) denomina "sistemas tecnológicos". Y aunque obviamente los contextos metalúrgicos prehistóricos no son equiparables sí puede resultar interesante pensar las metalurgias antiguas como "sistemas tecnológicos". Y tratando de hacerlo para el caso de la metalurgia del hierro en el Viejo Mundo (Humphris y Rehen, 2013; Wertime y Muhly, 1980) parece que la emergencia del hierro en Anatolia (Yalçin, 1998; Leek, 2014; Muhly et al., 1985) y su expansión por la cuenca del Mediterráneo (Pleiner, 1980; 1982a; 1982b; Snodgrass, 1980; Waldbaum, 1999) estuvo correlacionada con varios factores: 1) densidades de población alta, 2) ciudades y núcleos de población de cierta importancia y 3 ) la escritura y sistemas de pesos y medidas (Killick, 2015). Por otra parte, los estimulantes ensayos de Alexander $(1980,1983)$ explorando la expansión del hierro en distintos continentes con sugestivas ideas apenas han tenido continuidad en las últimas décadas (una excepción: Kim, 2001).
En todo caso, la arqueología, tradicionalmente, no ha prestado demasiada atención a los procesos de invención e innovación en general hasta hace poco tiempo (Fitzhugh, 2001; O’Brien y Shennan, 2010; Schiffer, 2011; Van der Leeuw, 2010).

En la metalurgia protohistórica cada fase del proceso de producción de hierro origina sus propios y exclusivos desechos además de otros elementos identificables en el registro arqueológico (Fig. 1). Desgraciadamente en la mayoría de los casos las escorias - de distintas clases - son los restos más visibles y en muchas ocasiones los únicos. El proceso de trabajo del hierro (Arnoldussen y Brusgaard, 2015) va desde la extracción -Fase 1- (búsqueda y extracción de minerales de hierro), a través de la fundición -Fase 2- (en los hornos se reduce el mineral a una "esponja" metálica) y la forja primaria -Fase III- (la "esponja” se transforma por martilleado en caliente en barras y pellas metálicas), hasta la forja secundaria -Fase 4- (martilleado en caliente y frío para convertir las barras/pellas en objetos de hierro). Lo ideal para un estudio paleometalúrgico es obviamente disponer de restos de la cadena operativa completa. Pero en el caso de la Península Ibérica para la etapa inicial de introducción del hierro y la Primera Edad del Hierro, que es el periodo considerado en este estudio, el conjunto de datos disponibles es muy limitado porque buena parte de la cadena operativa no está representada en el registro arqueológico disponible. En

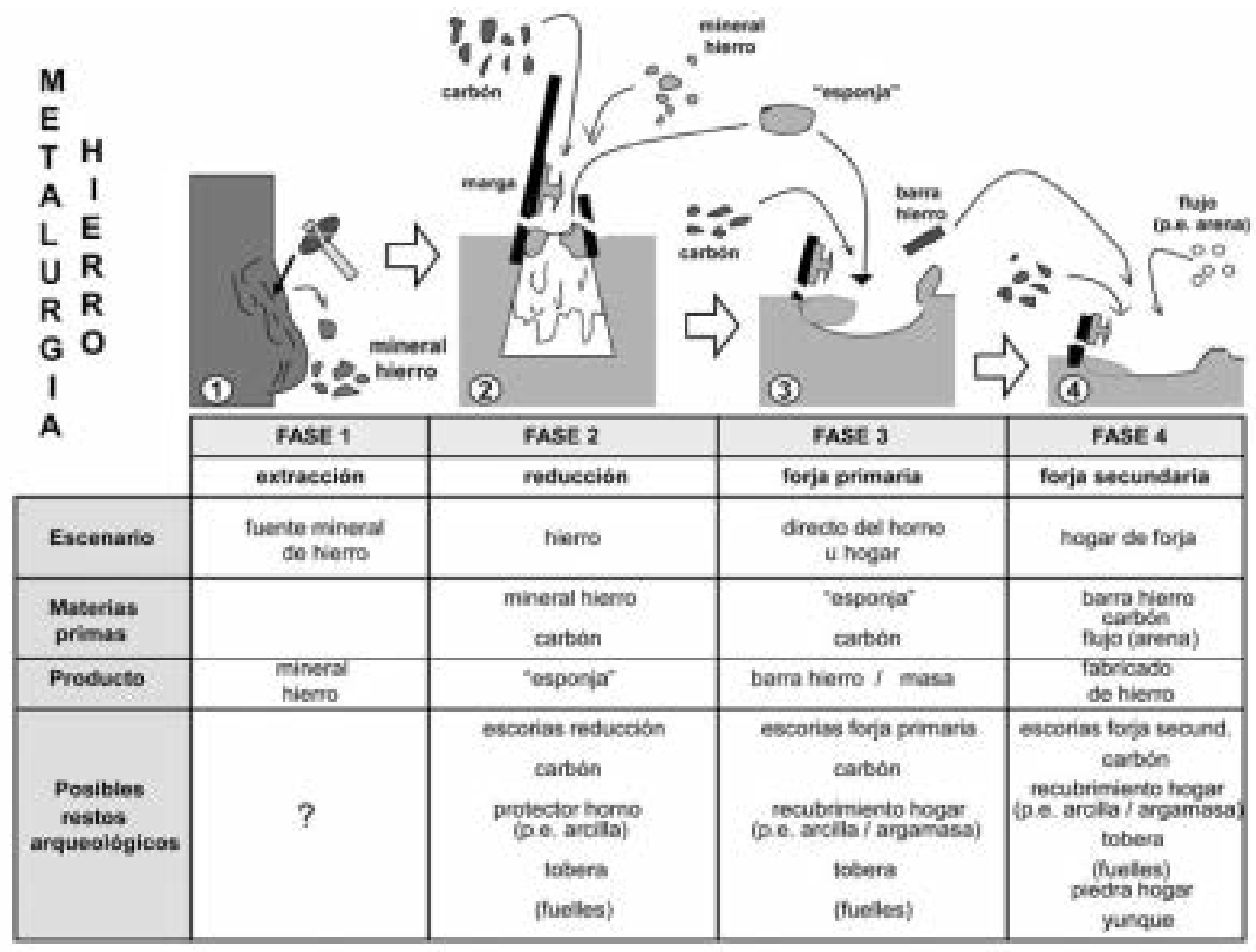

Figura 1. Las cuatro fases de la metalurgia de hierro: 1 (extracción), 2 (reducción), 3 (forja primaria) y 4 (forja secundaria), con la indicación de las materias primas, los productos resultantes y los posibles correlatos arqueológicos de cada fase (según Arnoldussen y Brusgaard 2015 modificado). 
consecuencia, el volumen y calidad de la información que tenemos es muy escaso.

De todas formas si se considera el número de sitios excavados de la Primera Edad del Hierro y las superficies exhumadas la conclusión contundente es que las evidencias materiales de la metalurgia de hierro son muy pobres. Si nos preguntamos por las causas de que las actividades metalúrgicas sean difíciles de descubrir podemos argumentar en distintas direcciones: 1) la peligrosidad de las actividades pirotecnológicas haría aconsejable situarlas en la periferia o fuera de los propios asentamientos, 2) las excavaciones generalmente se realizan en zonas centrales de los lugares de habitación y no en la periferia y zonas aledañas, 3) las estructuras de talleres metalúrgicos, especialmente los hornos, una vez abandonadas quedan muy alteradas y con poca evidencia aparente, 4) los materiales implicados en la metalurgia debieron ser reciclados continuamente y también amortizados fuera de sus contextos de producción y uso, y por último, 5) el desarrollo tardío de los estudios de arqueometalurgia del hierro que ha obligado durante mucho tiempo a disponer únicamente de los objetos de hierro y las escorias carentes de toda analítica.

En este estudio presentamos la situación de los primeros hallazgos de piezas de hierro en la Península Ibérica en contextos del Bronce Final y la distribución de los fabricados de hierro en la Primera Edad del Hierro. Se sugieren las condiciones de producción y los procesos de adopción con sus posibles vías de expansión. Insistimos en la necesidad de más estudios arqueometalúrgicos por un lado y por otro en la búsqueda de los contextos demográficos, sociales y políticos en los que se produce la innovación y desarrollo del trabajo del hierro. En última instancia, se trata de abrir los tradicionales límites conceptuales en los que se ha movido el tema y de cerrar los vacíos entre la dimensión tecnológica y sociológica de la metalurgia del hierro (Chirikare, 2015: 12).

\section{EL PRIMER HIERRO EN EL OCCIDENTE DE EUROPA}

La introducción del hierro en la Península Ibérica (Almagro Gorbea, 1993; Ruiz Zapatero et al., 2012) se atribuyó hasta finales de los años 70 del siglo XX a la influencia hallstáttica llegada desde el otro lado de los Pirineos en los siglos VIII-VII a.C. (Palol y Wattenberg, 1974). Ya en los años 80, las excavaciones en los asentamientos fenicios del Sur de España empezaron a vincular el primer hierro con la colonización fenicia (Aubet, 1986). Esta visión arribó con fuerza en los manuales de la época, aunque no faltaban propuestas más indigenistas, centradas sobre todo en las comunidades del Bronce Final, sus relaciones transpirenaicas el hierro ya era conocido en las regiones de Aquitania y el Languedoc desde el siglo VIII a.C.- y la influencia que sobre su desarrollo ejercerían sucesivamente fenicios y griegos (Junyent, 1992: 27). Finalmente, en la última década se ha comprobado que los objetos de hierro más antiguos se encuentran en contextos del Bronce Final (siglos XII-X a.C.) del Sur y Oeste peninsular, vinculados a la llamada "Precolonización" (AlmagroGorbea, 1993; Ruiz-Gálvez, 1993), esto es, a los contactos y navegaciones desde el Mediterráneo Oriental inmediatamente anteriores al establecimiento de los primeros asentamientos fenicios estables en las costas de Andalucia, alrededor del 825/800 a.C. (Celestino et al. 2008; González de Canales et al., 2004) (Fig. 2).

Los primeros objetos de hierro fechados por C-14 nos remiten a un marco temporal claramente precolonial, que transcurre desde la segunda mitad del siglo XII a.C. hasta los alrededores del año 1000 a.C. Por un lado, en la región de las Beiras (centro de Portugal) han aparecido cuchillos de dorso curvo y sierras de hierro en asentamientos como Monte do Trigo, Beijós, Moreirinha (Idanha-a-Nova) y Monte do Frade (Penamacor), con dataciones de los siglos XII-X a.C. (Vilaça, 2006). Por ahora deben considerarse los hierros más antiguos de Iberia. Recientemente Mederos (2008: 63-64, 2009) ha evaluado 14 cuchillos y 4 sierras de estos poblados del centro de Portugal, resaltando que son hierros "blandos", es decir, no superiores en dureza a un buen bronce. Se trata de cuchillos sacrificiales para dar muerte a animales que tienen sus prototipos en cuchillos chipriotas del Periodo Chipriota Final IIIA1 (12251150 a. C.). En el Sureste Peninsular, el depósito de Villena (Alicante) - un fabuloso tesoro con vajilla y brazaletes de oro y plata, entre otras piezas (Soler, 1965)incluye un brazalete de hierro y un remate de hierro con incrustaciones de oro, tal vez se trate del pomo de un cetro o espada, que resulta difícil de fechar con precisión pero que es anterior al 1100 a.C. y posiblemente se ocultara en torno a los siglos XIII-XII a.C. (Hernández et al., 2014). Es importante destacar su relación con el knowhow chipriota, como ha señalado Ruiz-Gálvez (1992 y 1993). Además, estamos ante un clarísimo ejemplo del valor del primer hierro como elemento exótico y suntuario en estos momentos tempranos: pieza bimetálica (con oro) asociada a joyas y vajilla de oro y plata.

Volviendo al centro de Portugal, en Nossa Senhora da Guia (Baiões, S. Pedro do Sul) se halló uno de los depósitos prehistóricos más extensos de la Edad del Bronce, más de 80 piezas con casi 18 kilos de metal, que reunía vajilla y elementos de banquete, armas y ostentosas piezas de exhibición militar, algunos objetos de adorno, vestimenta y cuidado corporal, así como distintas herramientas entre las que cabe destacar un escoplo o gubia de bronce con punta de hierro y un cuchillo también de hierro con enmangue de bronce (Silva et al., 1984: 170; Armbruster, 2002-2003: 146). Se ha discutido la procedencia de algunas de las piezas de bronce, como los carros con copa, tradicionalmente valorados como una influencia mediterránea en la Lusitania cen- 
tral durante la época del Bronce Final. Algún trabajo reciente muestra sin embargo de manera contundente su conexión con el centro de Europa, especialmente con la región meridional de los Alpes, hacia la cual hasta ahora se conocen muy pocos contactos arqueológicos (Schattner, 2011). Fechable alrededor del siglo IX a.C., el depósito incluye una gran mayoría de piezas de fabricación local y algunas importaciones mediterráneas precoloniales entre las que se encuentra el hierro, otra vez en objetos bimetálicos, algo por otro lado muy característico de los primeros objetos de hierro del Mediterráneo Oriental y Central (Almagro-Gorbea, 1993: 84-86; Ruiz-Gálvez, 1993: 49-52). Algún resto de hierro no determinable se ha documentado en el depósito de la Ría de Huelva (s. X-IX a.C.) (Ruiz-Gálvez, 1995: 137-138) que repite la aparición de algo de hierro como en muchos depósitos italianos del Bronce Final (Almagro-Gorbea, 1993: 87). En el poblado portugués de Sâo Juliao (Braga), alguna pieza de hierro podría ser ya de manufactura local dentro del s. IX a.C. (Bettencourt, 2000: 123). En cualquier caso, no parece desencaminada la idea de que a pesar de su exotismo en los contextos indígenas del Bronce Final, los pocos elementos de hierro -y otros elementos mediterráneos- no debieron producir un impacto profundo en la vida de las gentes del Occidente peninsular, más allá del círculo de las élites (Vilaça, 2012).

Otro caso de bimetalismo es el documentado en la sepultura 4 de La Encantada (Cuevas del Almanzora, Almería), en el Sureste peninsular. Se trata de un frag- mento informe constituido por una lámina de hierro revestida con restos de bronce $(91,76 \% \mathrm{Cu}, 6,88 \mathrm{Sn}$, 1,36 PB), posiblemente un útil similar al citado de Baiões, y fechado alrededor del siglo IX-inicios del VIII a.C. (Lorrio, 2008a: 86, 297-298; Montero Ruiz, 2008, 504). Otro hallazgo singular es un fragmento de un objeto de hierro indeterminado recuperado en una escombrera metalúrgica vinculada a la fase del Bronce Final del poblado de Peña Negra (González Prats, 1992: 245, 253-254), para la que cabe proponer una fecha del siglo IX a.C.

En el Occidente peninsular las evidencias más antiguas que poseemos serían el depósito hallado en un fondo de cabaña de El Berrueco (Salamanca), un poblado de finales de la Edad del Bronce, y una azuela de apéndices del mismo yacimiento (Morán, 1924: 22, lám. XIII,B; Maluquer, 1956 y 1958: 48, fig. 8). El depósito estaba formado por 2 brazaletes y 2 navajas de afeitar en bronce y 2 escoplos, un punzón y una anilla de hierro. Su presencia no supone a priori un dominio de la producción local, pero el hecho de que se trate de útiles conlleva cierto conocimiento de su funcionalidad. Las navajas se basan en prototipos atlánticos y mediterráneos de bronce, por lo que reflejan una primera toma de contacto con este metal (Almagro-Gorbea, 1993: 86 ss.). El depósito debe fecharse en el siglo IX a.C. y se explica por la importancia de la Vía de la Plata como eje de comunicación. Seguramente podría vincularse a un jerarca del poblado. Las navajas representan elementos de tocado personal que se identifican con un

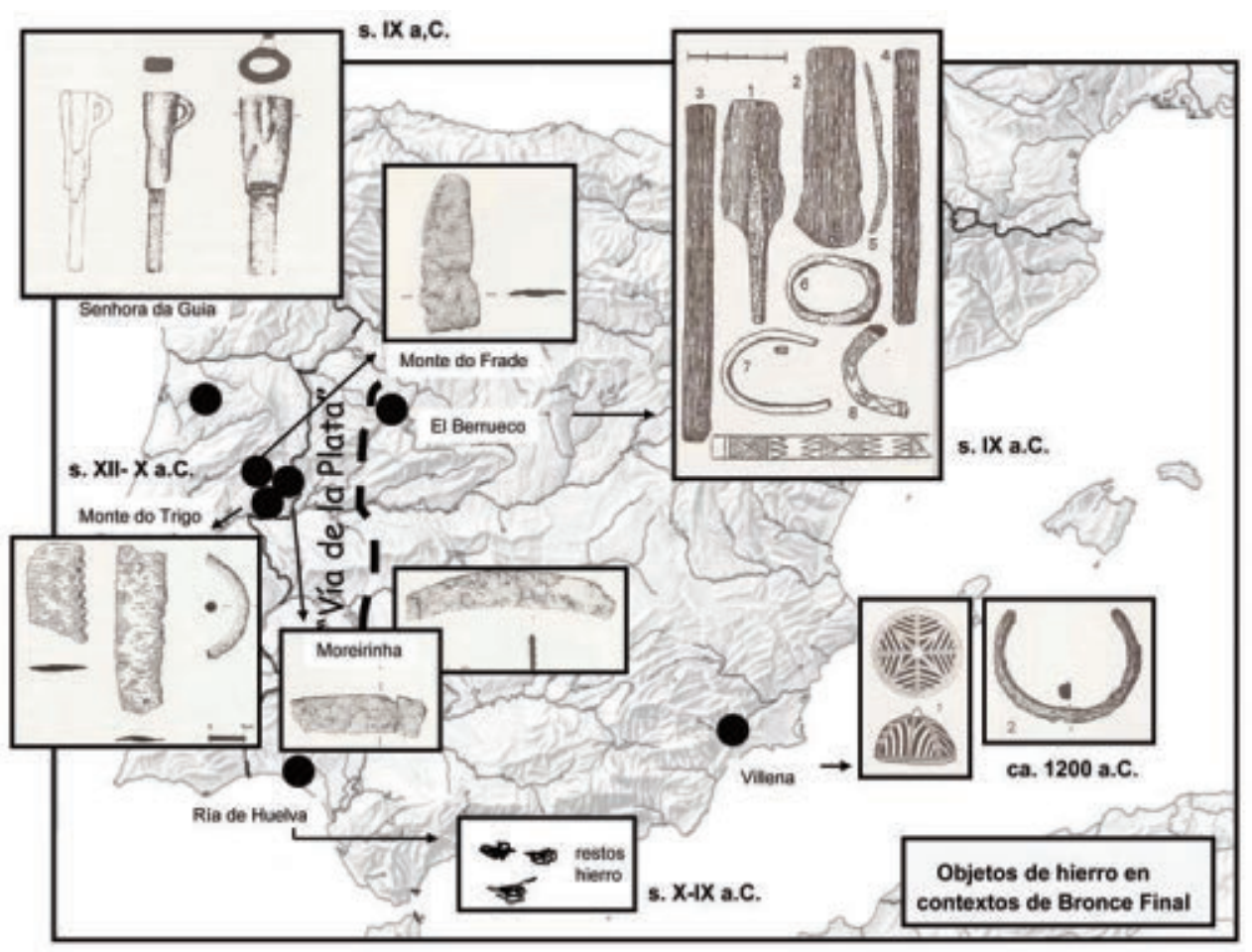

Figura 2. Mapa de distribución de los hallazgos de objetos de hierro en contextos de Bronce Final (según Ruiz Zapatero et al. 2012). 
patrón estético muy determinado -el cuidado de la barba-y que, como en el caso de los peines de marfil o las pinzas de depilar, constituirían un símbolo de edad y jerarquía (Goody, 1982; Ruiz-Gálvez, 1995: 139). Otro elemento muy interesante y que enlaza con lo anterior sería la azuela de apéndices de El Berrueco. La excepcionalidad de la pieza, al tratarse de un ejemplar de hierro, fue también valorada por Almagro-Gorbea (1993: 82-84) juntamente con las hachas del depósito de Campotéjar (Granada), que fecha en los siglos X-IX a.C. y cuyas tipologías relaciona con las piezas de bronce itálicas continentales y de Sicilia, que a su vez derivarían de prototipos del Mediterráneo oriental.

Un depósito de objetos simbólicos y preciosos recientemente hallado en la sevillana cámara megalítica de Palacio III (Almadén de la Plata) incluyó dos fragmentos de barra de hierro (Murillo-Barroso et al., 2015). En la ocultación se habían colocado también algunos objetos de cuarzo, cuentas de ámbar, un pendiente de plata, dos fusayolas y una aguja de bronce. Los análisis de algunos materiales indican la coexistencia de tecnologías locales y otras alóctonas relacionables con el ámbito colonial mediterráneo y una amortización intencional entre los s. IX y VI a.C., fruto sin duda de un momento de contacto colonial, simultáneamente expresando resistencia y asimilación cultural por parte de las comunidades indígenas.

Como hemos visto, el contexto de los primeros objetos de hierro aparece claramente vinculado al carácter del hierro como metal semiprecioso, exótico y suntuario, como revelan los depósitos de Villena y Baiões o los escondrijos de El Berrueco y Palacio III. Su conexión con las elites es clara y bien podría simbolizar el control de la producción metalúrgica por los individuos más sobresalientes. La posesión de piezas de hierro era un indicador de prestigio y este carácter debió guiar el interés posterior por el metal "negro".

\section{EXPANSIÓN Y DIFUSIÓN: LA COLONIZACIÓN FENICIA}

Las primeras factorías fenicias en las costas andaluzas se fechan en torno al último tercio del siglo IX a.C. (Aubet, 1986; 2001). En sus ocupaciones de finales del siglo VIII a.C. se documenta a su vez la primera producción de hierro, la primera siderurgia estable en la Península Ibérica (Delgado, 2008), tal y como ocurre en el Cerro del Villar (Aubet et al., 1999), Toscanos (Keesmann et al., 1983) y Morro de Mezquitilla en Málaga (Schubart, 1985). Sin olvidar los objetos de hierro precoloniales, hay que dejar claro que la introducción del trabajo del hierro en Iberia se debe a los agentes coloniales fenicios y que a partir de esta introducción el hierro será progresivamente adoptado por las comunidades indígenas más próximas (Ruiz Zapatero et al., 2012; Renzi et al., 2013: 179-180).
En Toscanos, Morro de Mezquitilla y Cerrro del Villar los restos de hornos documentados están muy arrasados y no puede determinarse su forma original. Pero aún así, no deja de resultar interesante la reciente observación de que la tecnología siderúrgica que conocemos -aunque con bastantes limitaciones- en las factorías fenicias del Sur y Sureste peninsular no incorpora toda la tecnología del trabajo del hierro que se conocía entonces en el Mediterráneo Oriental (Renzi et al., 2013: 185-6), aunque desconocemos la razón última de ese desequilibrio entre el trabajo de los herreros de las metrópolis fenicias y los de las colonias del Occidente mediterráneo.

Las primeras espadas de hierro que se conocen en el ámbito tartésico del sur de Iberia aparecen ya en el siglo VII a.C. (Torres, 2002: 264-265; Farnié y Quesada, 2005: 43-45, 52-57). Su diseño, más relacionado con la metalurgia atlántica de finales de la Edad del Bronce, en concreto las tradicionales espadas largas de doble filo, sugiere una invención local. Éste sería el caso de la única espada de hierro del tipo conocido como RondaSa-Idda hallada en una de las tumbas orientalizantes de Cástulo (Jaén), en el alto valle del Guadalquivir (Jiménez Ávila, 2002: 242). Lo cierto es que se conocen muy pocas espadas en tumbas aristocráticas en este momento (Almagro-Gorbea, 1992: nota 80), lo que al margen de las limitaciones tecnológicas que supone el forjado y que explicaría la excepcional presencia de estos objetos, podría relacionarse con la adopción de prácticas militares más propias del Próximo Oriente, donde los soldados de infantería no llevan espadas, siendo las armas más habituales la lanza y el arco (Torres, 2002: 265). A este respecto, resulta significativo que en el Suroeste peninsular lo que podríamos llamar como panoplia orientalizante se caracterice durante los siglos VII-VI a.C. por la presencia de largas puntas de lanza, que pueden llegar a superar los $50 \mathrm{~cm}$ de longitud, acompañadas de sus regatones y de cuchillos curvos, lo que sin duda debe verse como un claro indicio de prestigio social (Lorrio, 2008b; 2008c).

Los fenicios extendieron su influencia por las costas del Sureste de España y el Levante y alcanzaron las Islas Baleares, donde la fecha tradicional de la fundación histórica de Ebusus (Ibiza) hacia el 654 a. C. está ya desfasada, ya que la presencia fenicia en la zona se data con relativa seguridad entre finales del siglo VIII y comienzos del siglo VII a.C., como demuestra la fundación de Sa Caleta (Sant Josep de sa Talaia), en la costa suroccidental de la isla (Ramon Torres, 2007). La presencia fenicia en el Sureste está bien documentada desde mediados o la segunda mitad del siglo VIII a.C. con los asentamientos de La Fonteta y El Cabezo Pequeño del Estaño (Guardamar del Segura, Alicante), en la desembocadura del río Segura (Rouillard et al., 2007; González Prats, 2011; 2014; García y Prados, 2014).

La presencia de escorias en los niveles fundacionales de La Fonteta pertenecientes a Fonteta I y II, fecha- 
dos entre mediados o la segunda mitad del siglo VIII y el primer tercio del VII a.C., permiten señalar el temprano desarrollo del trabajo metalúrgico del hierro, elemental y poco refinado en la tecnología aplicada en la combustión, vinculada a la identificación de hornos de pozo con presencia de restos de escorias en su interior (Renzi y Rovira, 2005: 169-170; González Prats, 2010: 45-48, 68, 82), observándose una evolución hacia hornos más complejos, documentados a lo largo de los siglos VII a.C. y VI a.C. en el propio enclave deLa Fonteta a partir de su fase III (González Prats, 2014: 35-36) y en El Castellar de Librilla III y IV, en Murcia, con el denominado horno E (Ros Sala, 1993: 96; véase una crítica en Gómez, 1999: 120). Los hallazgo de Fonteta son la evidencia de la primera tecnología productiva siderúrgica desarrollada en el Sureste, que implica una explotación de minerales férricos muy puros, en relación con una obtención de filones o minerales muy superficiales, que dejan poca evidencia visible en el registro arqueológico (Renzi y Rovira, 2015: 124).

Las poblaciones indígenas del Sureste adoptaron rápidamente la metalurgia de hierro como prueban los hallazgos de la fase orientalizante de Peña Negra (Crevillent, Alicante), aunque hasta la fecha no se hayan detectado evidencias de actividad siderúrgica en este destacado yacimiento (González Prats, 2005: 805), sí documentados en cambio en El Castellar de Librilla
(Ros Sala, 1993), mientras que en Vinarragell (Castellón), un fragmento de hierro se sitúa en un nivel anterior a la aparición de las cerámicas fenicias (Clausell, 2004). Un dato muy interesante se encuentra en el cementerio de Les Moreres, vinculado al núcleo urbano de Peña Negra. En la tumba 42 de esta necrópolis, perteneciente a un varón adulto, un cuchillo de hierro se asocia a una fíbula de doble resorte, así como a una urna de tipo Cruz del Negro de producción local, que cabe fechar en la segunda mitad del siglo VII avanzado (Torres, 2008: 641). Por su parte, en la tumba 73, de una mujer adulta joven con un neonato, se recuperaron dos plaquitas de hierro se depositaron junto a otra fíbula de doble resorte. Son las únicas fíbulas de este tipo en el cementerio y se asocian a los pocos objetos de hierro allí recuperados (González Prats, 2002: 275). La asociación cuchillo de dorso + fíbula de doble resorte se va a repetir en muchos otros casos de la primera penetración del hierro hacia el interior de Iberia y, sin duda alguna, constituyen en origen dos elementos de identidad. El origen de este tipo de fíbula ha sido muy discutido, tanto en su variante más oriental -a partir del comercio fenicio y jonio-cretense (Almagro Basch, 1966: 222 ss.) -como central- en la Italia continental y Sicilia (Cuadrado, 1963: 24). Ambas posturas han sido objeto de recientes críticas a partir de criterios cronotipológicos, concluyendo un origen autóctono aunque inspiradas en modelos sicilianos e italianos (Torres,

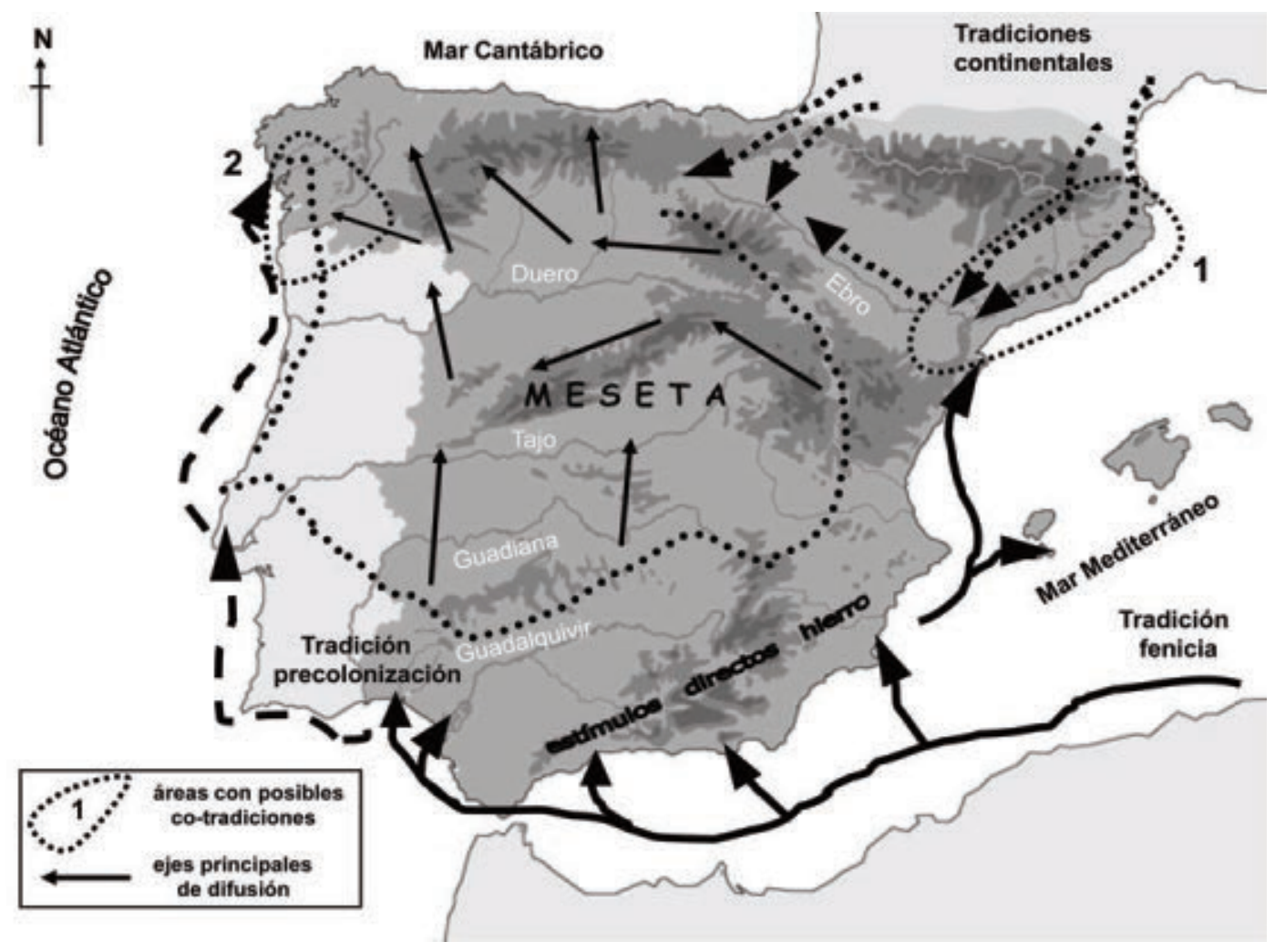

Figura 3. Mapa con las tradiciones culturales portadoras de la metalurgia de hierro en la Península Ibérica y los principales ejes de expansión. 
2002: 196; Almagro-Gorbea, 2008: 531). Por otra parte, el catálogo de fíbulas de doble resorte en el Golfo de León Occidental, al sur de Francia, supera el centenar de ejemplares en contextos en los que no hay indicios de presencia fenicia, reforzando su atribución a un origen local (Graells, 2014), aunque la "inspiración” mediterránea de este tipo de fíbula parece clara.

El estudio de los primeros objetos de hierro del Noreste peninsular se ha abordado tradicionalmente desde la óptica tipológica y sólo muy puntualmente desde la arqueométrica (Rovira, 2010). Los resultados aportados por algunos yacimientos, como las necrópolis de incineración de Can Piteu-Can Roqueta (Barcelona), Agullana (Gerona) y el Molar (Tarragona), nos permiten avanzar su arranque hasta como mínimo el año 700 a.C. (López Cachero et al., 2009; Toledo y Palol, 2006; Rafel y Armada, 2008). Incluyen cuchillos de tipo Gand Bassin I, anillas, navajas de afeitar, asadores de hierro y fíbulas de doble resorte. Nuevas dataciones radiocarbónicas inducen a una cierta prudencia y sugieren incluso una corrección al alza en medio siglo de las fechas tradicionales atribuidas a la última de estas necrópolis (Rafel y Armada, 2008: 151 ss.).

Es interesante destacar el estudio que se realizo sobre los distintos ritmos de difusión del hierro y las cerámicas en el área catalana (Ruiz Zapatero, 1992a). El hierro, por su mayor atracción y capacidad de difusión, se extendió con más rapidez y a mucha mayor distancia que las cerámicas de importación. En algún caso sabemos que un jerarca local, como en Aldovesta (Bajo Ebro), controlaba la distribución de la mercadería fenicia hacia las poblaciones del interior (Mascort et al., 1991). Aquí, en un pequeño hábitat defendido y ocupado brevemente entre la segunda mitad del siglo VII a.C. y el primer cuarto del VI a.C., se hallaron más de un centenar de ánforas fenicias, dos cuchillos de hierro seguramente relacionados con el sacrificio de animales y la distribución de comida, e indicios de fundición y trabajo del metal en otro de los sectores del poblado (Sardá, 2010: 486 ss.).

En Els Vilars (Arbeca, Lérida), hacia el interior, en un poblado fortificado se ha documentado el desarrollo de una metalurgia del hierro en torno al año 800-775 a.C. (GIP, 2003: 234, 264-266). Seguramente se trata de la residencia de un cabecilla local o bien un grupo militar que controla la explotación económica de un territorio organizado en pequeños asentamientos. Se ha sugerido que ésta sea el resultado de influencias del otro lado de los Pirineos, al igual que las armas - espadas de antenas y puntas de lan$\mathrm{za}$ - de las necrópolis de la región del Ampurdán (Junyent, 1992: 21-35). En todo caso, estas posibles influencias de la metalurgia del hierro de la zona del Sureste de Francia fueron más tardías que las del Sur de Cataluña, ligadas al comercio fenicio y mucho más limitadas (Armada et al., 2005; López Cachero, 2007). La escasez de análisis metalográficos no permite por ahora precisar más este debate entre los influjos continentales y los mediterráneos para el primer hierro en el Noreste de Iberia (Ruiz Zapatero y Rovira, 1996; Rovira, 2010). Desde el Bajo Ebro los conocimientos de la metalurgia del hierro remontaron el curso del río y ya en Cortes de Navarra aparecen tempranamente en la segunda mitad del s. VII a.C. (véase Fig. 3).

De manera que un doble acervo parece estar presente en el origen del armamento de hierro en el siglo VII a.C. en el Suroeste y Noreste de la Península Ibérica (Farnié y Quesada, 2005: 216). Las espadas de antenas y otras armas señalan influencias norpirenaicas, dadas las intensas relaciones entre el Noreste peninsular y el Sur de Francia, mientras que las espadas de lengüeta y botón terminal remiten a una tradición meridional que se remonta al final de la Edad del Bronce, con espadas largas de doble filo, que gradualmente darán paso a otras más cortas características de la cultura ibérica (falcatas), seguramente relacionadas con una nueva forma de combatir.

Otro factor clave para comprender la aparición y difusión del hierro en la Península Ibérica es el descubrimiento, durante los últimos años, de que los navegantes fenicios llegaron al Sur de Portugal y remontaron la costa hasta el Sur de Galicia (Arruda, 1999-2000; Pellicer, 2000). En el siglo IX a.C. se fechan las primeras ocupaciones fenicias en el Algarve y el Bajo Tajo, y a finales de esa centuria los fenicios llegan a Galicia buscando estaño, oro y tal vez también esclavos. Como en el Noreste, los primeros hierros deben vincularse a la presencia fenicia en la zona (Fig. 3). Las piezas más antiguas, en torno a los siglos VIII-VII a.C., han aparecido en los castros de Torroso y Penalba, en Pontevedra (Álvarez Núñez, 1991; Peña, 1992: 38-39). Los cuchillos afalcatados podrían ser importaciones, pero las hoces que imitan tipos atlánticos en bronce deben ser producciones locales (González Ruibal, 2006-2007: 244). Pequeños fragmentos se conocen también en Castrovite y Neixón Pequeno. Junto a los cuchillos, como en Cataluña, se difundieron las fíbulas de doble resorte. Es verdad que los objetos de hierro se conservan muy mal en los suelos ácidos de Galicia y que los minerales de hierro son relativamente escasos en la región; y lo cierto es que el número total de piezas de hierro conocidas es pequeño. En todo caso, el hierro llegó en fechas antiguas y se debió extender con rapidez por el Noroeste, a pesar de que no se encuentre en algunas excavaciones de castros. En la Primera Edad del Hierro el nuevo metal se empleó en armas cortas - puñales - y lanzas que reflejan un tipo de combate comunitario, no-aristocrático (González Ruibal, 2006-2007: 226-232).

\section{EL PRIMER HIERRO EN EL INTERIOR PENINSULAR}

Como hemos visto, la introducción inicial del hierro en Iberia fue debida a los agentes coloniales fenicios (Renzi et al., 2013: 179-81), realizándose a lo largo de un amplio frente costero que desde las costas meridionales se extendió progresivamente por el Noreste hasta Cataluña y por el Noroeste hasta Galicia (AlmagroGorbea, 1993). Desde todas estas áreas la metalurgia de 
hierro fue rápidamente penetrando hacia el interior (Fig. 3). En el siglo VII a.C. el trabajo del hierro está bien atestiguado por todo el Sur peninsular. Y tanto en estas áreas como en el Levante será la base de la excelente metalurgia de los pueblos iberos, desarrollada a partir del s. VI a.C. Por su parte, en la gran plataforma central de Iberia, la Meseta, el trabajo del hierro debió seguir tres vías principales:

1) Desde el Levante peninsular (Arenas, 1999) y el Valle del Ebro (Ruiz Zapatero, 1992b) se introdujo en el Oriente de la Meseta a finales del s. VII a.C. y posiblemente desde algo antes. Cuchillos de dorso y fíbulas de doble resorte serían algunos de los primeros objetos introducidos (Lorrio, 1997: 152 ss.), aunque en los últimos años se haya identificado también la presencia de algunas espadas en necrópolis de las provincias de Soria y Guadalajara (Lorrio, 2016). La producción de hierro, favorecida en unos casos por la proximidad de importantes afloramientos en el Sistema Ibérico, permitió desarrollar desde el principio un eficaz instrumental. Una vez aprendidas las técnicas de fundir y forjar el hierro, muchas comunidades sacaron ventaja del metal que tenían en su territorio (Ruiz Zapatero et al., 2012). El hierro fue especialmente importante para las armas, ya presentes en los primeros cementerios celtibéricos, sin antecedentes en las tradiciones metalúrgicas locales.

2) Desde el Sureste los contactos fueron intensos aunque el registro arqueológico de la Meseta Sur es bastante más pobre (Ruiz Zapatero, 2007: 47 ss.) y carecemos de argumentos para justificar si la presencia de objetos es debida a la importación de los mismos, a la comercialización de materia prima en bruto (lingotes) o a la adopción de los conocimientos necesarios para obtener y trabajar el hierro (Montero Ruiz y Renzi, 2012: 342). Aunque el proceso de introducción del hierro se produce a lo largo de este primer milenio a.C., es sorprendente la falta de datos sobre sus primeros testimonios, incluso en periodos como la segunda Edad del Hierro en los que la siderurgia ya está consolidada (Torres, 2013).

3) Finalmente, la vía quizás más importante fue la del sur, siguiendo la llamada Vía de la Plata y buscando los vados del río Tajo (Álvarez-Sanchís, 2000: 70-71, fig. 3). La distribución de los objetos de hierro más antiguos y de las fíbulas de doble resorte, jalona hacia el interior la difusión de éstos y otros elementos orientales como jarros y braseros de bronce, servicios de vajilla, y joyas entre otras cosas. De este modo puede seguirse perfectamente el camino de penetración del comercio tartésico hasta Cáceres y las altas tierras de Ávila y Salamanca.

Así, en la tumba orientalizante de El Carpio (Toledo) se hallaron dos cuchillos de hierro asociados a un exótico y rico ajuar de origen marcadamente oriental (una clepsidra para trasvasar líquidos, un vaso de plata para libaciones, jarritas fenicias de aceite perfumado) correspondiente a una mujer de alto estatus que se fecha alrededor del año 650 a.C. (Pereira, 1989). Y en otro vado del mismo río, en Talavera la Vieja (Cáceres), se han identificado posibles lingotes de hierro en contextos funerarios datados a finales del s. VII a.C. (Jiménez Ávila, 2006: 106-108). No obstante, las difíciles comunicaciones desde el área nuclear tartésica hacia Extremadura han matizado esta última interpretación a favor de un eje oeste-este, siguiendo las cuencas del SadoGuadiana, Tajo y Mondego (Arruda, 2000; Pereira, 2008: 121).

La publicación de las fechas de C-14 de la necrópolis de Palomar de Pintado (Villafranca de los Caballeros, Toledo) ha venido a elevar significativamente la datación de los primeros objetos de hierro en la región (Pereira et al., 2003: 162). Una de las tumbas de incineración de la fase más antigua del cementerio deparó un ajuar formado por un brazalete de bronce y un cuchillo afalcatado de hierro que se ha fechado a comienzos del siglo IX a.C. Ello le convierte en uno de los más antiguos de la Meseta Sur, seguramente junto con el escoplo de la Muela de Alarilla (Guadalajara) (Méndez y Velasco, 1986: 28) y la pieza de la tumba 32 de Arroyo Culebro (Leganés, Madrid) (Penedo et al., 2001), que cuenta con una fecha de termoluminiscencia que eleva su cronología hasta el siglo IX a.C. (Ruiz Zapatero, 2007: 49-55). Cuchillos de hierro de dorso ligeramente curvo están presentes en la necrópolis de Arroyo Butarque (Madrid) a finales del siglo VII o inicios del VI a.C. (Blasco et al., 2007), junto a otros elementos como fíbulas de doble resorte y colgantes amorcillados. Estos objetos entroncan el cementerio con un ambiente orientalizante y advierten de la influencia que esos círculos pudieron tener en la introducción de los ritos de cremación en estas áreas del interior peninsular.

En la Meseta occidental y el valle medio del Duero contamos con otras dataciones antiguas, en torno a los siglos IX-VIII a.C., aunque en contextos mucho más pobres y sin apenas analíticas. Se trata de cuchillos, navajas de afeitar, escoplos, punzones, hachas y azuelas, que implican un cierto conocimiento de su funcionalidad y tecnología, tal vez asociado a la figura del especialista itinerante (Álvarez-Sanchís y Ruiz Zapatero, 2014: 208-209). Por ejemplo, en el Castillo de Burgos se fecha escoria de hierro en el s. IX a.C. (Junyent, 1992: 27), en el Soto de Medinilla (Valladolid) aparecen restos informes en un nivel fechado radiométricamente en el 725 a. C. (Delibes et al., 1995: 174), y del castro de Sanchorreja (Ávila) proceden dos pequeños cuchillos de hierro que, por su relación con las cerámicas halladas, podrían llevarse al siglo VIII a.C. (González-Tablas, 1986-87: 51). Del mismo sitio se conocen varias hachas de hierro con alerones o apéndices laterales incipientes (González-Tablas et al., 1991-92: 309, 325-326), que tendrían su precedente inmediato en las hachas del Bronce Final. Más al oeste, en Ledesma (Salamanca), algunos fragmentos de hierro, entre ellos 
una hoja y un pequeño cincel, se datan en la primera mitad del s. VII a.C. (Benet et al., 1991: 130). Escorias y un fragmento de tobera de fuelle halladas en Herguijuela de Ciudad Rodrigo (Salamanca) atestiguan también una siderurgia local en fechas tempranas (López Jiménez y Benet, 2005: 1022, fig. 6). A partir del siglo VI a.C. los objetos de hierro se van generalizando en los asentamientos de la cultura de El Soto (Romero y Ramírez, 1996; Celis 2002). No obstante, la plena tecnología del trabajo del hierro no se producirá hasta después del 500 a.C.

Por último, en el Norte de Iberia, la región cantábrica, la introducción del hierro no debió tener el desfase cronológico o retraso tradicionalmente asumido, si bien es cierto que las evidencias firmes no parecen ser anteriores a finales del siglo VII o ya del VI a.C. (Fanjul y Marín, 2006: 114 ss., 123). En Asturias, una varilla de hierro de Camoca (Camino, 1995: 122), un punzón de Campa Torres (Maya y Cuesta, 2001: 76-77, 146) y algunos fragmentos informes de Picu Castiellu de Moriyón (Camino, 1992: 140), se fechan en el s. VI a. C. De esa fecha o algo anterior pueden ser las hojas de puñal de Os Castros de Taramundi y Chao Sanmarti (Camino y Villa, 2014: 61-62). En Cantabria y en fechas parecidas se deben situar las escorias de hierro y un posible horno de reducción del castro de Castilnegro (Valle y Serna, 2003: 363, 376), así como los restos de escorias del castro de La Garma (Pereda, 1999: 76). En cualquier caso son muy escasas evidencias para el número de sitios excavados y no hay prueba alguna de extracción minera ni reducción de mineral en ningún asentamiento (Camino y Villa, 2014).

\section{LA BÚSQUEDA DE CONTEXTOS PARA LA METALURGIA DE HIERRO}

La idea de Snodgrass (1980) de medir la expansión valorando la proporción de objetos de hierro y bronce en las distintas etapas de transición (Fig. 4), debería ser llevada a cabo detalladamente en distintas escalas, pero sobre todo a escala regional. Los resultados son muy relevantes, como bien demostró Waldbaum para el Mediterráneo Occidental (1980 y 1999). La reciente propuesta de Needham (2007), identificando tres modelos: el de la transición gradual, el de la adopción rápida del hierro y el denominado "la crisis del bronce", es una herramienta más sofisticada pero en la misma dirección. Porque, sobre todo, hay que convenir en que la clave explicativa debe buscarse en el uso social y el significado del metal en cada sociedad. La transición metalúrgica entre el Bronce Final y la Primera Edad del Hierro es un proceso complejo y no debe ser valorado como una mera sustitución del bronce por el hierro, sino como un desplazamiento del sistema de valor social de cada época. El hierro fue, únicamente, un factor más en la transición a la Edad del Hierro.
La secuencia que podemos establecer para Iberia es la siguiente. En primer lugar, una fase pionera o introductoria del hierro, en la que primero aparecen pocos objetos de este metal - en buena medida a modo de importaciones - y luego comienza un trabajo local del hierro a pequeña escala, coexistiendo con la metalurgia del bronce. A continuación sigue una etapa de crecimiento y difusión de la metalurgia del hierro, así como de diversificación de los tipos fabricados. Por último viene la producción de hierro a gran escala, con el desarrollo pleno del utillaje agrícola y presumiblemente con mejoras tecnológicas como la carburización de los filos (Fig. 5).

Pero la necesidad de ampliar y mejorar los contextos de ese proceso dibujado de forma muy general e imprecisa, de cuantificar los datos y detallar las cronologías es muy grande. Además la hegemonía del paradigma histórico-cultural en la Arqueología de la Edad del Hierro española se explica, en buena medida, porqué tenemos todavía sólo un cuadro muy general con bastantes lagunas. Pero, sobre todo, este paradigma no ha creado nuevas preguntas sobre la significación social, económica y política del hierro (Giles, 2007; Needham, 2007). Incluso podemos afirmar que el tema no es un objetivo específico de investigación en nuestro país (Ruiz Zapatero, 2007: 38).

La posibilidad de considerar, como sugirió hace años uno de nosotros (Ruiz Zapatero, 1992a), que el modelo de disponibilidad de Zvelebil y Rowley-Conwy (1986) pueda ser adaptado para el fenómeno de la difusión del hierro creemos que merece una reconsideración. También deberíamos esforzarnos en buscar las huellas que pueden dejar los herreros itinerantes en el registro arqueológico, aunque resulte ciertamente una tarea difícil (Neipert, 2006).

Por otro lado, necesitamos superar planteamientos sociales básicamente economicistas como los sugeridos por Childe (1942), Driehaus (1965), Frankenstein y Rowlands (1978) o Wells (1980), sobre si el hierro democratizó o aceleró el proceso de jerarquización social, para pasar a establecer análisis más contextuales y completos. Un ejemplo es la reciente sugerencia de Giles (2007) de considerar el trabajo del hierro una metáfora del poder y la autoridad social, y explorar sus posibles dimensiones simbólicas y las formas de transmisión de los conocimientos especializados que implica su metalurgia (Fig. 6).

\section{A MODO DE CONCLUSIONES}

El estudio de la metalurgia del hierro en la Protohistoria europea se ha desarrollado de forma notable en las dos últimas décadas (Hosek et al., 2011; Pleiner, 2000; Waldbaum, 1999; Gener Moret, 2014), pero al mismo tiempo parece que los especialistas en arqueometalurgia han formado una especie de gueto con sus propios 


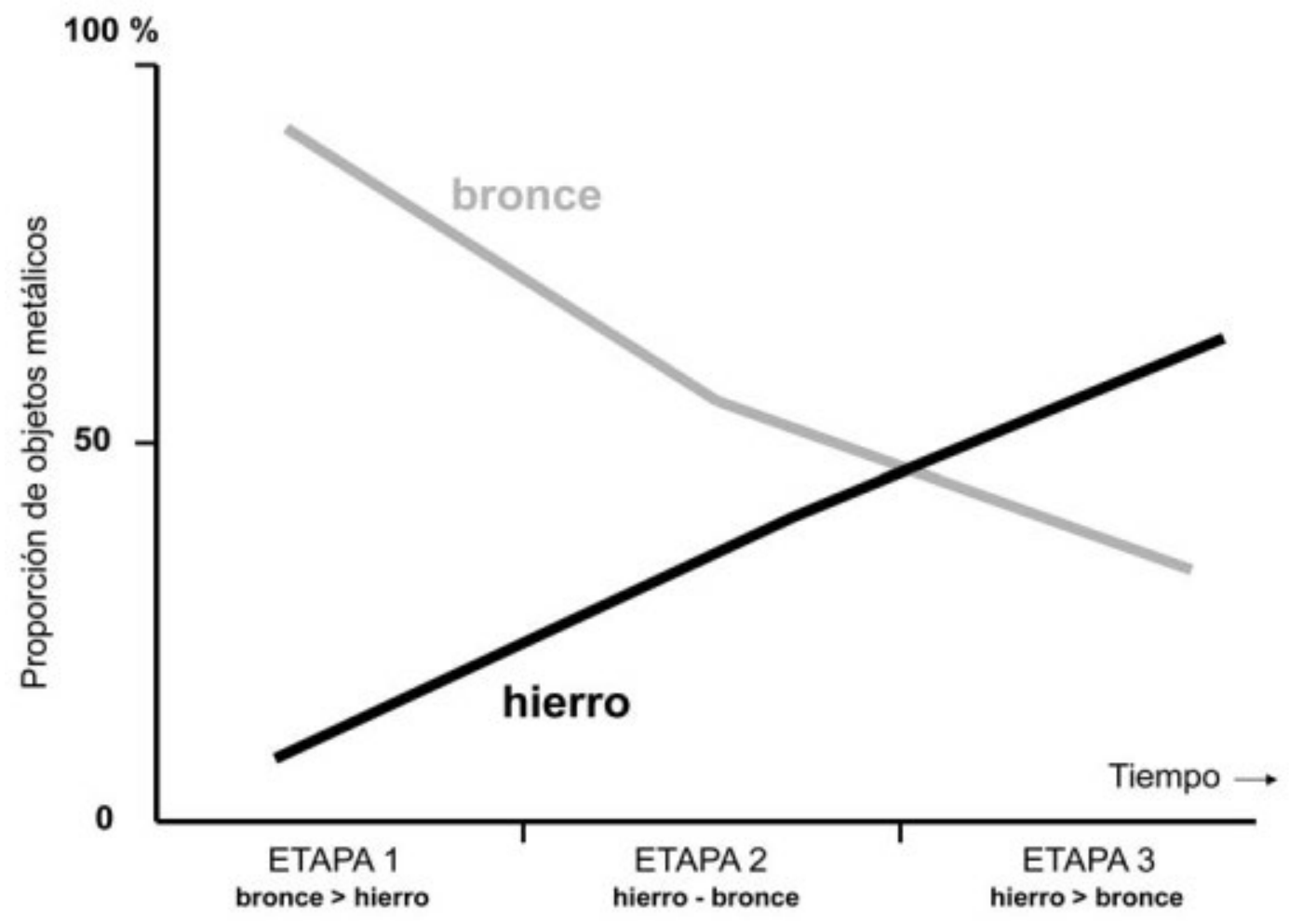

Figura 4. Las fases de la implantación de la metalurgia de hierro en relación con la del bronce (según datos de Snodgrass 1980).
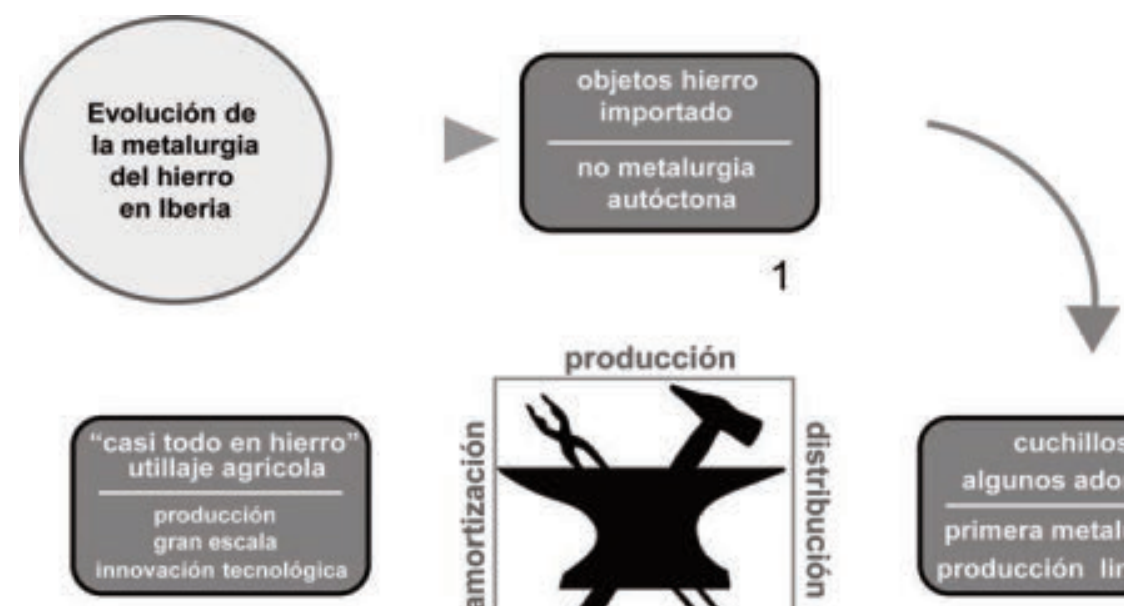

4
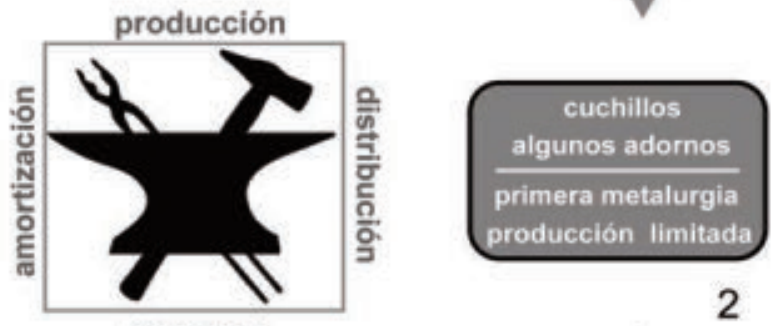

2
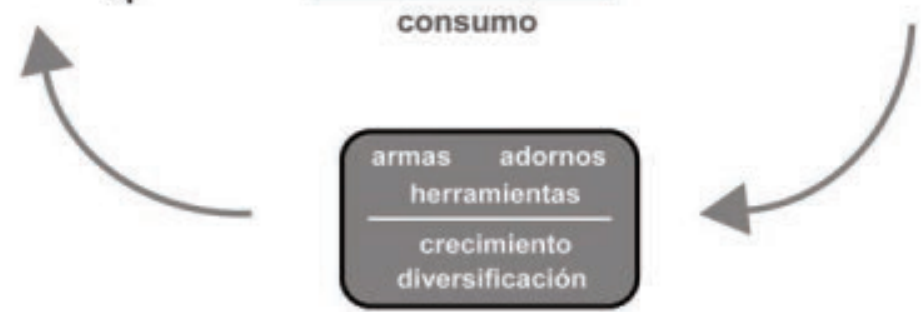

3

Figura 5. Características generales de la evolución de la metalurgia de hierro en la Península Ibérica según etapas de implantación. 


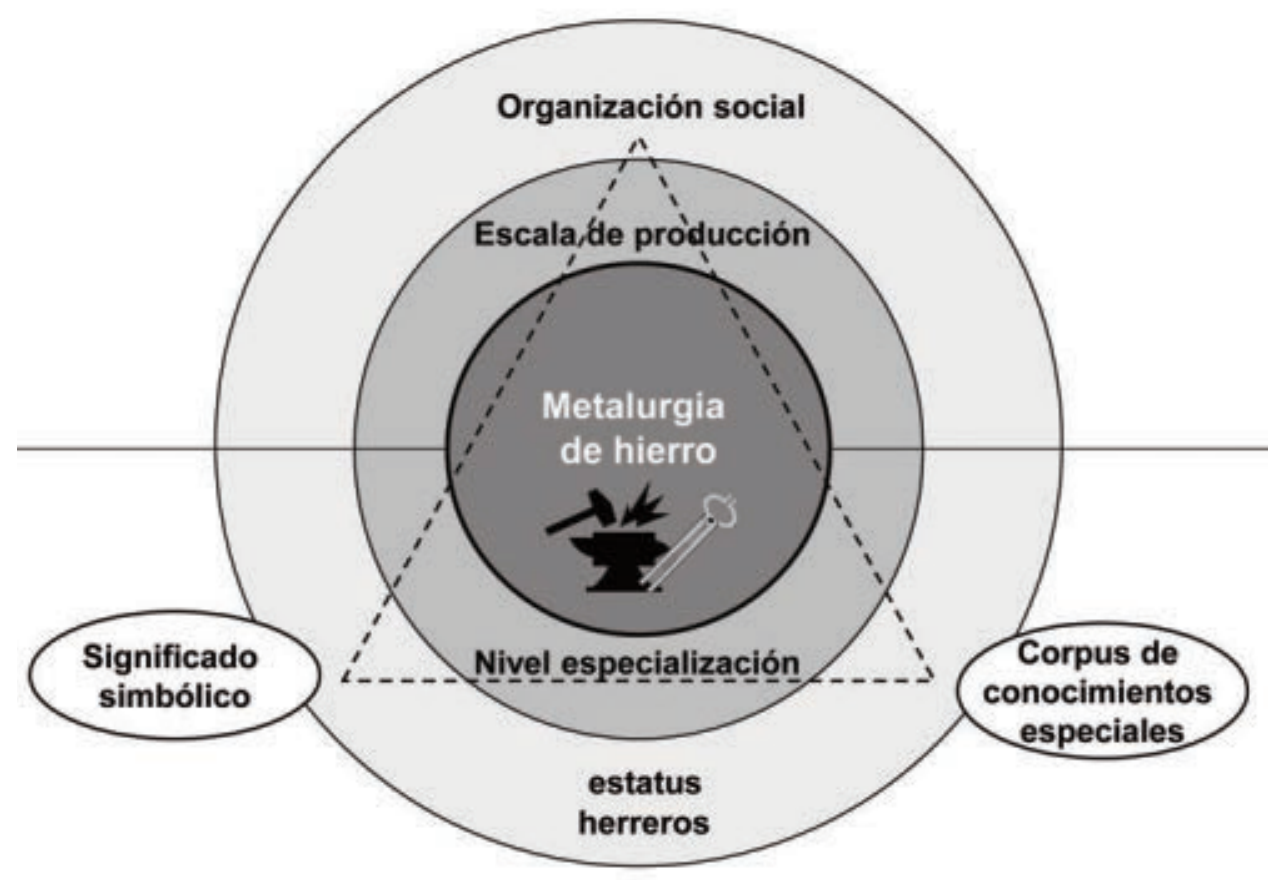

Figura 6. Matriz de contextualización de la metalurgia de hierro (en parte según datos de Giles 2007).

congresos y revistas especializadas pero con débil presencia en las principales revistas internacionales (Killick, 2015: 298). El hecho de que algunos especialistas seniors no tuvieran formación arqueológica específica puede ayudar, en parte, a entender la situación actual. De forma que reivindicar la "arqueometalurgia como arqueología" (Killick, 2015) tiene bastante sentido si queremos ampliar e insertar la antigua metalurgia en los problemas generales de las comunidades de la Edad del Hierro, para así "seguir golpeando mientras el hierro este caliente" en feliz expresión de Quirós Castillo (2014: 10).

Las ideas simplistas del difusionismo más exacerbado como única explicación para la expansión de la tecnología del hierro están superadas por datos de diversas regiones del Viejo Mundo. Y la metalurgia del hierro no tuvo un solo origen o área nuclear (Alpern, 2005) como parece que tampoco se pueda seguir sosteniendo la idea difusionista de que el trabajo del hierro se desarrolló a partir de la metalurgia del cobre (Bocoum, 2004: 28). Como tampoco parece cierto que la adopción del hierro se impusiera rápidamente y se extendiera como mancha de aceite por regiones extensas. Tenemos evidencias claras de que la expansión de la metalurgia del hierro no fue siempre homogénea y rápida sino más bien gradual e inconstante con expansiones "en mosaico" y desfases cronológicos importantes entre áreas próximas como sucede en la región cos- tera del Mediterráneo Oriental (Gottlieb, 2010).

Parece evidente que en la actualidad el panorama que tenemos para la introducción de la primera metalurgia del hierro en Iberia adolece de bastantes limitaciones que podemos resumir así:

1) No disponemos de datos sobre los lugares y formas de extracción de minerales de hierro en prácticamente ninguna de las áreas peninsulares. Tampoco conocemos su relación espacial con los asentamientos.

2) Casi lo mismo se puede decir de los sitios y contextos de producción del metal, las herrerías. Y contando con las superficies de excavaciones en área en algunos poblados las estructuras localizadas están claramente descompensadas: los hornos, talleres y espacios de trabajo del hierro son casi invisibles.

3) Es imposible evaluar los volúmenes de producción de hierro aunque la impresión es que los fabricados de hierro fueron escasos en asentamientos y enterramientos. Desconocemos casi por completo la visibilidad real del nuevo metal en la vida cotidiana de las comunidades del Hierro durante los primeros compases.

4) Aún con todas las limitaciones la impresión general es que la producción de hierro fue una tarea de ámbito doméstico, como tantas otras de la Primera Edad del Hierro: procesado de alimento, fabricación cerámica, elaboración de tejidos y otras de menor importancia. Pero hasta que punto sólo ciertos indivi- 
duos conocían los secretos de la metalurgia y como se articulaban éstos socialmente es algo que solo podemos elucubrar.

5) No disponemos de ratios de objetos de bronce versus objetos de hierro en cada sitio por fases y por tanto tampoco a nivel regional, una cuantificación básica para comprender los ritmos y factores implicados en la adopción del metal negro.

6) El número de análisis arqueométricos para determinar rasgos tecnológicos del proceso metalúrgico son bastante escasos, tanto referidos a analíticas de escorias, de residuos de producción como de objetos de hierro. Especialmente en lo que se refiere a la determinación del momento de implantación de los conocimientos de la carburización para el endurecimiento de filos.

7) Los análisis de uso, duración, reparación y amortización de los objetos de hierro son virtualmente inexistentes, ya que carecemos de las denominadas "biografías de objetos".

8) Desconocemos la dimensión simbólica y significación ritual que debió asociarse a los herreros, como se deduce de estudios etnoarqueólogicos e históricos (Giles, 2007; Haaland, 2007-8; Hingley, 1997), pues dominar el hierro significa controlar la transformación de minerales en armas, de las que depende la defensa y el ataque, y en herramientas que aseguran la subsistencia. Lo que sugiere una ambigüedad del metal asociado al nacimiento y fertilidad por un lado y a la destrucción y la muerte por otro. Y en todo ello la etnoarqueología del hierro fuera de Europa también puede resultar de ayuda (Haaland, 2006).

\section{BiBLIOGRAFÍA}

Alexander, J. (1980): "The spread and development of iron-using in Europe and Africa", en Leakey, R.E. y Ogot B.A. (eds.): Proceedings of the 8th Pan African Congress on Prehistory and Quaternary Studies, Nairobi 1977. Nairobi, International Louis Leakey Memorial Institute for African Prehistory: 327-330.

Alexander, J. (1983): "Some neglected factors in the Spread of iron-using in Europe". Offa, 40: 29-33.

Almagro Basch, M. (1966): "Sobre el origen posible de las más antiguas fíbulas anulares hispánicas”. Ampurias, 28: 215-236.

Almagro-Gorbea, M. (1993): "La introducción del hierro en la Península Ibérica. Contactos precoloniales en el periodo Protoorientalizante". Complutum, 4: 81-94.

Almagro-Gorbea, M. (2008): La Necrópolis de Medellín. II. Estudio de los hallazgos. Bibliotheca Archaeologica Hispana 26-2. Real Academia de la Historia, Madrid.
Alpern, S. B. (2005): “Did They or Didn't They Invent it? Iron in Sub-Saharan Africa". History in Africa, 32: 41-94. http://dx.doi.org/10.1353/hia.2005.0003

Álvarez Núñez, A. (1991): "Castro de Penalba (Campo Lameiro, Pontevedra)". Arqueoloxía/Informes, 2: 37-42.

Álvarez-Sanchís, J. R. (2000): “The iron age in western Spain (800 BC-AD 50): an overview". Oxford Journal of Archaeology, 19 (1): $65-89$. http://dx.doi.org/10.1111/1468-0092.00100

Álvarez-Sanchís, J. R. y Ruiz Zapatero, G. (2014): "The Emergence of Urbanism in Early Iron Age Central Iberia", en Fernández-Götz, M., Wendling H. y Winger, K. (eds.): Paths to Complexity. Centralisation and Urbanisation in Iron Age Europe. Oxbow Books, Oxford: 204-213.

Arenas, J. (1999): “Contactos entre el Oriente meseteño y Levante en los albores de la Edad del Hierro", en Villar F. y Beltrán F. (eds.): Pueblos, Lenguas y Escrituras en la Hispania Prerromana. VII Coloquio sobre Lenguas y Culturas Paleohispánicas (Zaragoza 1998). Universidad de Salamanca: 75-90.

Armada, X.L.; Garcia y Rubert, D.; Montero, I.; Moreno I.; Rafel, N. y Rovira, M.C. (2005): "Minería y metalurgia durante la I Edad del Hierro. Procesos de cambio en el sur de Cataluña". Revista d'Arqueologia de Ponent, 15: 133-150.

Armbruster, B. (2002-2003): “A metalurgia da Idade do Bronze Final Atlântico do Castro de Nossa Senhora da Guia, de Baioes (S. Pedro do Sul, Viseu)". Estudos Pré-históricos, 10-11: 145-155.

Arnoldussen, S. y Brusgaard, N. O. (2015): "Production in deposition: structured deposition of Iron Age ironworking elements (The Netherlands)". LUNULA. Archaeologia protohistorica, XXIII: 115-124.

Arruda, A.M. (1999-2000): Los fenicios en Portugal: Fenicios y mundo indígena en el centro y sur de Portugal (siglos VIII-VI a.C.). Cuadernos de Arqueología Mediterránea 5-6. Universidad Pompeu Fabra. Barcelona.

Aubet, $M^{a}$.E. (1986): "Los fenicios en España: estado de la cuestión y perspectivas", en G. Del Olmo y $\mathrm{M}^{\mathrm{a}}$.E. Aubet (eds.): Los fenicios en la Península Ibérica. I. Ed. Ausa, Sabadell: 149-175.

Aubet, Mª. E. (2001): The Phoenicians and the West: Politics, Colonies and Trade. Cambridge.

Aubet, $M^{\text {a }}$.E.; Carmona, P.; Curià, E.; Delgado, A.; Fernández, A. y Párraga, M. (1999): Cerro del Villar-I: el asentamiento fenicio en la desembocadura del río Guadalhorce y su interacción con el hinterland. Arqueología Monografías. Junta de Andalucía, Sevilla.

Benet, N.; Jiménez, M.C. y Rodríguez, M.B. (1991): "Arqueología en Ledesma, una primera aproximación: la excavación en la plaza de San Martín”, en 
M. Santonja (coord.): Del Paleolítico a la Historia. Junta de Castilla y León. Museo de Salamanca: 117136.

Bettencourt, A. M. (2000): O povoado de Sâo Juliao, Vila Verde, Norte de Portugal, nos finais da Idade do Bronze e na transiçâo para a Idade do Ferro. Instituto de Ciencias Sociais, Cadernos de Arqueologia 10. Braga.

Blasco, C.; Barrio, J. y Pineda, P. (2007): "La revitalización de los ritos de enterramiento y la implantación de las necrópolis de incineración en la cuenca del Manzanares: la necrópolis de Arroyo Butarque", en A. Dávila (ed.): Estudios sobre la Edad del Hierro en la Carpetania. Museo Arqueológico Regional, Alcalá de Henares: Zona Arqueológica 10, vol II: 216-238.

Bocoum, H. Ed. (2004): The Origins of Iron Metallurgy in Africa. New light on its antiquity: West and Central Africa. Paris, UNESCO.

Camino, J. (1992): “Excavaciones arqueológicas en castros de la Ría de Villaviciosa: un poblamiento de la Edad del Hierro". Excavaciones Arqueológicas en Asturias 1987-1990, 2: 137-144.

Camino, J. (1995): “Excavaciones arqueológicas en los castros de Villaviciosa: apuntes para una sistematización de la Edad del Hierro". Excavaciones Arqueológicas en Asturias, 3: 117-126.

Camino, J. y Villa, A. (2014): "El hierro en el registro arqueológico de la protohistoria cantábrica”. Kobie Anejo 13: 59-74.

Celestino, S., Rafel, N. \& Armada, X.-L. Eds. (2008): Contacto cultural entre el Mediterráneo y el Atlántico (siglos XII-VIII ane): la precolonización a debate. Madrid: Consejo Superior de Investigaciones Científicas (Escuela Española de Historia y Arqueología en Roma, Serie Arqueológica 11).

Celis, J. (2002): “El Bronce Final y la Primera Edad del Hierro en el Noroeste de la Meseta", en M.A. Blas Cortina y A. Villa Valdés (eds.): Los poblados fortificados del NO de la Península Ibérica: formación y desarrollo de la cultura castreña. Navia: 97-126.

Childe, V.G. (1942): What Happened in History. Penguin Books.

Clausell, G. (2004): "El Torrelló del Boverot: del bronce medio al comienzo del hierro". Actas del Congreso La Edad del Bronce en tierras valencianas y zonas limitrofes (Villena 2003). Villena: 167-176.

Cuadrado, E. (1966): "Precedentes y prototipos de la fíbula anular hispánica". Trabajos de Prehistoria, 7.

Delgado, A. (2008): "Fenicios en Iberia", en F. Gracia (coord.): De Iberia a Hispania. Ariel-Prehistoria, Barcelona: 347-474.

Delibes, G.; Romero, F. y Ramírez, Ma.L. (1995): “El poblado «céltico» de El Soto de Medinilla (Valladolid). Sondeo estratigráfico de 1989-1990", en G. Delibes, F. Romero y A. Morales (eds.): Arqueolo- gía y Medio Ambiente. El primer milenio a.C. en el Duero medio. Junta de Castilla y León. Valladolid: 149-177.

Driehaus, J. (1965): "Fürstengräber" und Eisenerze zwischen Mittelrhein, Mosel und Saar". Germania, 43: 32-49.

Fanjul, A. y Marín, C. (2006): "La metalurgia del hierro en la Asturias castreña: nuevos datos y estado de la cuestión”. Trabajos de Prehistoria, 63 (1): 113-131.

Farnié, C. y Quesada, F. (2005): Espadas de hierro, grebas de bronce. Símbolos de poder e instrumentos de guerra a comienzos de la Edad del Hierro en la Península Ibérica. Monografías del Museo de Arte Ibérico de El Cigarralejo, Murcia.

Fitzhugh, B. (2001): "Risk and invention in human technological evolution”. Journal of Anthropological Archaeology, 20: 125-167. http://dx.doi.org/10.1006/jaar.2001.0380

Frankenstein, S. y Rowlands, M.J. (1978): “The internal structure and regional context of Early Iron Age society in south-western Germany". Bulletin Institute of Archaeology, 15: 73-112.

García, A. y Prados, F. (2014): “La presencia fenicia en la Península Ibérica: el Cabezo Pequeño del Estaño (Guardamar del Segura, Alicante)". Trabajos de Prehistoria, 71 (1): $113-133$. http://dx.doi.org/10.3989/tp.2014.12127

Gener Moret, M. (2014): "Una aproximación a la tecnología del hierro prehidráulico y su estudio". Kobie Serie Anejo, 13: 13-20.

Giles, M. (2007): "Making metal and forging relations: ironworking in the British Iron Age". Oxford Journal of Archaeology, 26 (4): 395-413. http://dx.doi. org/10.1111/j.1468-0092.2007.00290.x

Gómez, P. (1999): Obtención de metales en la Prehistoria de la Península Ibérica, BAR International Series, 753, Oxford.

González de Canales, F.; Serrano, L. y Llompart, J. (2004): El emporio fenicio precolonial de Huelva (ca. 900-770 a.C.). Biblioteca Nueva. Madrid.

González Prats, A. (1992): "Una vivienda metalúrgica en la Peña Negra (Crevillente-Alicante). Aportacion al conocimiento del Bronce Atlántico en la Península Ibérica". Trabajos de Prehistoria 49: 243-257.

González Prats, A. (2002): La necrópolis de cremación de Les Moreres (Crevillente, Alicante, España) (s. IX-VII a.C.). Universidad de Alicante. Alicante.

González Prats, A., 2005: "El fenómeno orientalizante en el Sudeste de la Península Ibérica”, en S. Celestino y J. Jiménes (eds.): El periodo orientalizante, Actas del III Simposio Internacional de Arqueología de Mérida: Protohistoria del Mediterráneo Occidental, Anejos de Archivo Español de Arqueología, XXXV: 799-808. 
González Prats, A. (2011): La Fonteta. Excavaciones de 1996-2002 en la colonia fenicia de la actual desembocadura del río Segura (Guardarmar de Segura, Alicante), Vol. 1. Seminarios Internaciones sobre Temas Fenicios, Alicante.

González Prats, A. (2014): La Fonteta-2. Estudio de los materiales arqueológicos hallados en la colonia fenicia de la actual desembocadura del río Segura (Guardamar del Segura, Alicante), tomos 1 y 2, Seminarios Internaciones sobre Temas Fenicios, Alicante.

González Ruibal, A. (2006-2007): Galaicos. Poder y comunidad en el Noroeste de la Península Ibérica (1200 a.C.-50 d.C.). Brigantium 18-19, 2 vols., A Coruña.

Goody, J. (1982): Cooking, cuisine and class. Cambridge Univ. Press. Cambridge.

Gottlieb, Y. (2010): "The Advent of the Age of Iron in the Land of Israel: A Review and Reassessment". Tel Aviv, 37: 89-110. http://dx.doi.org/10.1179/033443510x1263207017 9469

Graells, R. (2014): "Problemas de cultura material: las fibulas itálicas de la primera edad del hierro en el Golfo de León Occidenta"l. Madrider Mitteilungen, 55: 212-315.

GIP, Grup d'Investigació Prehistòrica (2003): "Caballos y hierro. El campo frisio y la fortaleza de "Els Vilars d'Arbeca" (Lleida, España), siglos VIII-IV a.n.e", en N. Alonso, E. Junyent, A. Lafuente y J.B. López (coords.): Chevaux de frise i fortificació en la primera edat del ferro europea. Lleida: 322-274.

Haaland, R. (2006): "Iron in the making: technology and symbolism. Ethnographic perspectives on European iron working”, en Andrén, A., Jennbert, K. y Randvere, C. (eds.): Old Norse religion in longterm perspectives. Origins, changes, and interactions, Lund, Nordic Academic Press : 79-85.

Haaland, R. (2007-2008): "Say it in Iron. Symbols of Transformation and Reproduction in the European Iron Age". Current Swedish Archaeology, 15-16: 91-110.

Haselgrove, C. y Pope, R. (eds.) (2006): The Earlier Iron Age in Britain and the Near Continent. Oxford, Oxbow.

Henrich, J. y Gil-White, F.J. (2007):" Why societies vary in their rates of innovation The evolution of innovation-enhancing institutions". http://www2.psych.ubc.ca/ henrich/Website/Paper s/ nventionInnovation05.pdf (Consultado 20-042016).

Hernández, M., García, G. y Barciela, V. (2014): “The treasures of Villena and Cabezo Redondo (Alicante, Spain)", en H. Meller, R. Ricch y E. Pernicka (eds.): Metalle der Macht - Frühes Gold und Silver,
Tagungen des Landesmuseums für $r$ Vorgeschichte Halle 11, Halle, (Saale): 593-607.

Hill, J.D. (1995): "The Pre-Roman Iron Age in Britain and Ireland (ca. 800 BC to AD 100): an Overview". Journal of World Prehistory, 9 (1):47-98.

Hingley R. (1997): "Iron, ironworking and regeneration: a study of the symbolic meaning of metalworking in Iron Age Britain", en A. Gwilt, and C. Haselgrove (eds.): Reconstructing Iron Age Societies. Oxford: Oxbow Books: 9-18.

Hošek, J.; Cleere, H. and Mihok, L. (eds.) (2011): The Archaeometallurgy of Iron. Recent Developments in Archaeological and Scientific Research. Archeologický ústav AVÈR, Praha.

Hughes, T. P. (1989): American Genesis: A Century of Invention and Technological Enthusiasm, 18701970. Nueva York, Viking Press.

Humphris, J. y Rehen, Th. (eds.) (2013): The World of Iron. Archetype.

Jiménez Ávila, J. (2002): La toréutica orientalizante en la Península Ibérica. Bibliotheca Archaeologica Hispana, 16. Real Academia de la Historia, Madrid.

Jiménez Ávila, J. (2006): “Los objetos de bronce y hierro", en J. Jiménez Ávila (ed.): El conjunto orientalizante de Talavera la Vieja (Cáceres). Memorias 5, Museo de Cáceres: 89-108.

Junyent, E. (1992): “Els orígens del ferro a Catalunya”. Revista d'Arqueologia de Ponent, 2: 21-35.

Keesmann, I.; Niemeyer, H.G. y Golschani, F. (1983): "Schlackenfunde von Toscanos". Madrider Mitteilungen, 24: 65-75.

Killick, D. (2015): "Invention and Innovation in African Iron-smelting Technologies". Cambridge Archaeological Journal, 25 (1): 307-319. http://dx.doi.org/10.1017/S0959774314001176

Kim, J. (2001): "Elite Strategies and the Spread of Technological Innovation: The Spread of Iron in the Bronze Age Societies of Denmark and Southern Korea". Journal of Anthropological Archaeology, 20 (4): 442-478. http://dx.doi.org/10.1006/jaar. 2001.0386

Leek, R. (2014): The Development of Iron Technology in the Mediterranean Bronze Age. Department od Archaeology, Conservation and History, University of Oslo. https://www.duo.uio.no/bitstream/handle/10852/40717/Leek_Master_Archaeology.pdf?s equence=1 (Consultado 21-04-2016).

López Cachero, F. J. (2007):" Sociedad y economía durante el Bronce Final y la Primera Edad del Hierro en el Noreste Peninsular: una aproximación a partir de las evidencias arqueológicas". Trabajos de Prehistoria, 64 (1): 99-120.

López Cachero, F. J., Rovira, M. C., Carlús, X., Lara, C. y Villena, N. (2009): «Nouvelles donnés concernant la transition entre le Bronze Final et le Premier Age 
du Fer en Catalogne côtiére : la nècropole à incineration de Can Piteu-Can Roqueta (Barcelone, Espagne)», en M-J. Lambert-Roulière, A. Daubigney, P.-Y. Milcent, M. Talon y J. Vital (eds.): De l'âge du Bronze a l'âge du Fer en France et en Europe Occidentale (X-VII siécle av. J-C.). Actes du XXX Colloque International de l'Association Française pour l'Étude de l'Age du Fer (AFEAF), Saint Romain-en-Gal-2006. Revue Archeologique de l'Est, 27 supp. Dijon: 213-226.

López Jiménez, O. y Benet, N. (2005): “Frontera y margen en el ámbito orientalizante: procesos históricos en la zona sudoccidental de la Meseta Norte", en S. Celestino y J. Jiménez (eds.): El Período Orientalizante. Actas del III Simposio Internacional de Arqueología de Mérida: Protohistoria del Mediterráneo Occidental. Anejos de Archivo Español de Arqueología XXXV: 1015-1024.

Lorrio, A.J. (1997): Los Celtíberos. Complutum Extra 7. Universidad de Alicante - Universidad Complutense de Madrid. Alicante.

Lorrio, A.J. (2008a): Qurénima. El Bronce Final del Sureste de la Península Ibérica. Bibliotheca Archaeologica Hispana 27. Real Academia de la Historia, Madrid.

Lorrio, A.J. (2008b): "Puntas de lanza", en M. Almagro-Gorbea (dir.): La necrópolis de Medellín. II. Estudio de los hallazgos, Bibliotheca Archaeologica Hispana, 26-2, Madrid: 563-565.

Lorrio, A.J. (2008c): “Cuchillos”, en M. Almagro-Gorbea (dir.): La necrópolis de Medellín. II. Estudio de los hallazgos, Bibliotheca Archaeologica Hispana, 26-2, Madrid: 566-571.

Maddin, R. (1982): "Early Iron Technology in Cyprus", en J. D. Muhlv, R. Maddin y V. Karagheorgis (eds.): Early Metallurgy in Cyprus, 4000-500 B. C. Larnaka: 303-313.

Maluquer, J. (1956): “La técnica de incrustación de Boquique y la dualidad de tradiciones cerámicas en la Meseta durante la Edad del Hierro". Zephyrus, VII: $179-206$

Maluquer, J. (1958): Excavaciones Arqueológicas en el Cerro del Berrueco (Salamanca), Acta Salmanticensia XIV-1. Salamanca.

Mascort, M.T.; Sanmartí, J. y Santacana, J. (1991): El jaciment protohistòric d'Aldovesta (Benifallet) $i$ el comerç fenici arcaic a la Catalunya meridional. Publicacions de la Diputació de Tarragona. Tarragona.

Maya, J.L. y Cuesta, F. Eds. (2001): El castro de la Campa Torres. Período Prerromano. VTP Editorial, Gijón.

Mederos, A. (2008): "El Bronce Final”, en F. Gracia (coord.): De Iberia a Hispania, Ariel, Barcelona: 19-91.
Mederos, A. (2009): "Metal para los dioses. La secuencia del grupo Baioes durante el Bronce Final II y el comercio Chipriota de hierro hacia Portugal (12001050 a.C.", en M. J Cruz Auñon y E. Ferrer (eds.): Estudios de Prehistoria y Arqueología en homenaje a Pilar Acosta Martínez. Universidad de Sevilla. Sevilla: 279-304.

Méndez, A. y Velasco, F. (1986): “Alarilla. Una propuesta de metodología arqueológica”. Arqueología espacial 9. Coloquio sobre el microespacio, Teruel: 17-32.

Montero Ruiz, I. (2008): “Apéndice I: Ajuares metalúrgicos y aspectos tecnológicos en la metalurgia del Bronce Final-Hierro en el Sudeste de la Península Ibérica", en A.J. Lorrio: Qurénima. El Bronce Final del Sureste de la Península Ibérica. Bibliotheca Archaeologica Hispana 27. Real Academia de la Historia, Madrid: 499-516.

Montero-Ruiz, I y Renzi, M. (2012): "Metalurgia en la Meseta Sur: síntesis sobre el primer milenio A.C.", en J. Morín de Pablos y D. Urbina (eds.): El primer milenio a.C. en la Meseta Central. De la longhouse al oppidum, Vol. I. Primera Edad del Hierro. Audema, Madrid: 341-350.

Morán, C. (1924): “Excavaciones Arqueológicas en el Cerro del Berrueco (Medinilla, Avila, El Tejado y Puente de Congosto, Salamanca)". Junta Superior de Excavaciones y Antigüedades, 65: 3-24 y XIV láms.

Mulhy, J.D.; Maddin, R.; Stech, T.; Özgen, E. (1985): "Iron in Anatolia and the Nature of the Hittite Iron Industry". Anatolian Studies, XXXV: 67-84.

Murillo-Barroso, M., Martinón-Torres, M., García Sanjuán,L., Wheatly, D., Hunt Ortiz, M.A., Fortaleza González, M. y Hernández Amedo, Mª J. (2015): "New objects in old structures. The Iron Age hoard of the Palacio III megalithic funerary complex (Almadén de la Plata, Seville, Spain)". Journal of Archaeological Science, 57: 322-344. http://dx.doi.org/10.1016/j.jas.2015.03.013

Needham, S. (2007): "800 BC, The Great Divide", en C. Haselgrove y R. Pope (eds.): The Earlier Iron Age in Britain and the Near Continent. Oxbow Books, Oxford: 39-63.

Neipert, M. (2006): Der Wanderhandwerker: Archäologisch-ethnografische Untersuchungen. Tübinger Texte 6, Rahden/Westf.

O’Brien, M. J. y Shennan, S. J. Eds. (2010): Innovations in Cultural Systems: Contributions from Evolutionary Anthropology. MIT Press, Cambridge, Mass.

Palol, P. de y Wattenberg, F. (1974): Carta Arqueológica de España. Valladolid. Valladolid.

Pellicer, M. (2000): "El proceso orientalizante en el occidente ibérico". Huelva Arqueológica, 16: 91134. 
Penedo, E.; Sánchez, M.; Martín, D. y Gómez, E. (2001): "La necrópolis de incineración de la Primera Edad del Hierro en el Arroyo Culebro (Leganés)", en Vida y Muerte en Arroyo Culebro (Leganés). Museo Arqueológico Regional. Madrid: 45-70.

Peña, A. de la (1992): Castro de Torroso (Mos, Pontevedra). Sintesis de las memorias de las campañas de excavaciones 1984-1990. Arqueoloxía-Memorias 11. Xunta de Galicia, A Coruña.

Pereda, E. (1999): "El alto de la Garma: un castro de la Edad del Hierro en el Bajo Miera". Regio Cantabrorum. Santander: 73-77.

Pereira, J. (1989): "Nuevos datos para la valoración del hinterland tartéssico. El enterramiento de la Casa del Carpio (Belvís de la Jara)", en Ma .E. Aubet (coord.): Tartessos. Arqueología Protohistórica del Bajo Guadalquivir. Sabadell: 395-409.

Pereira, J. (2008): "La tumba de casa del Carpio y el comercio en el valle del Tajo", en J. Álvarez-Sanchís (ed.): Arqueología Vettona. La Meseta Occidental en la Edad del Hierro. Zona Arqueológica, 12. Alcalá de Henares: 114-124.

Pereira, J.; Ruiz Taboada, A. y Carrobles, J. (2003): "Aportaciones del C-14 al mundo funerario carpetano. La necrópolis de Palomar de Pintado". Trabajos de Prehistoria, 60 (2): 153-168.

Pleiner, R. (1980): "Early Iron Metallurgy in Europe", en Th. A. Wertime y D. Muhly (eds.): The Coming of the Age of Iron, New Haven-Londres: 375-415.

Pleiner, R. (1982a): "Untersuchungen zur frühen Eisenverhüttang”. Das Altertum, 28 (1): 49-57.

Pleiner, R. (1982b): "Les débuts du fer en Europe". Dialogues de Histoire Ancienne, 8: 167-192.

Pleiner, R. (1997): "Les premieres produccions de ferro a l'Europa Central i Oriental'. Cota Zero, 13: 71-84.

Pleiner, R. (2000): Iron in Archaeology. The Europan Bloomeries Smelters. Praga. Ed. Archeologicky. USTAV AV CR.

Quirós Castillo, J. A. (2014): “Golpeando mientras el hierro esté caliente. Paleosiderurgia en el Norte peninsular”. Kobie Serie Anejo, 13: 5-12.

Rafel, N. y Armada, X-L. (2008): "Sobre la cronología de la necròpolis del Calvari del Molar i l'horitzó funerari del Bronce Final - Primera Edat del Ferro a l'Ebre. Noves datacions absolutes". Cypsela, 17: 149-159.

Ramon Torres, J. (2007): Excavaciones arqueológicas en el asentamiento fenicio de Sa Caleta (Ibiza). Ediciones Bellaterra.

Renfrew, C. (1978): “The anatomy of innovation", en D. Green, Haselgrove, C. y M. Spriggs, M. (eds.): Social organization and settlement. Oxford. BAR International Series Supplement, 47 (I): 89-117.

Renfrew, C. (1984): Approaches to Social Archaeo$\log y$. Edinburgh, Edinburgh University Press.

Renzi, M. (2013): La metalurgia del yacimiento fenicio de La Fonteta (Guardamar del Segura, Alicante). Bibliotheca Praehistorica Hispana, XXIX. Consejo Superior de Investigaciones Científicas, Madrid.

Renzi, M. y Rovira, S. (2005): "Escorias metalúrgicas del yacimiento fenicio de La Fonteta (Alicante). Estudio preliminar". VI Congreso Ibérico de Arqueometría, Madrid: 163-171.

Renzi, M. y Rovira, S. (2015): "Las metalurgias fenicias mediterráneas", en J.M. López Ballesta (coord.): PHICARIA, III Encuentros Internacionales del Mediterráneo. Minería y metalurgia en el Mediterráneo y su periferia oceánica, Mazarrón (Murcia): 113- 129.

Renzi, M.; Rovira, S.; Rovira, M. C. y Montero-Ruiz, I. (2013): "Questioning research on early iron in the Mediterranean", en J. Humphris y Th. Rehren, (eds.): The World of Iron, Archetype Publications, London: 178-187.

Romero, F. y Ramírez, Mª.L. (1996): "La Cultura del Soto. Reflexiones sobre los contactos entre el Duero medio y las tierras del sur peninsular durante la Primera Edad del Hierro", en M M $^{\mathrm{a}}$ A. Querol y T. Chapa (eds.): Homenaje al Profesor Manuel Fernández-Miranda. Complutum Extra 6, I: 313-326.

Ros Sala, Mª.M. (1993): "El trabajo del hierro en el poblado protohistórico de El Castellar (Murcia) I: análisis arqueológico", en S. Ramallo, A.M . Muñoz Amilibia, M ${ }^{a}$.M. Ros Sala y R. Arana (coords.): Metalurgia en la península Ibérica durante el primer milenio a.C.: estado actual de la investigación. Universidad de Murcia: 71-110.

Rouillard, P.; Gailledrat, E. y Sala, F. (2007): L'etablissement protohistorique de La Fonteta (fin VIIIe-fin Vie siècle av.J.-C.), Casa de Velázquez, 96, Madrid.

Rovira, M. C. (2010): “Tecnología de las primeras manufacturas férricas en el Noreste de la Península Ibérica". VII Congreso Ibérico de Arqueometría, Madrid: 458-467.

Ruiz-Gálvez, M. (1992): “La novia vendida: orfebrería, herencia y agricultura en la protohistoria de la Península Ibérica”. SPAL, 1: 219-251. http://dx.doi.org/10.12795/spal.1992.i1.11

Ruiz-Gálvez, M. (1993): “El occidente de la Península Ibérica, punto de encuentro entre el Mediterráneo y el Atlántico a fines de la Edad del Bronce". Complutum, 4: 41-68.

Ruiz-Gálvez, M. (1995): “El significado de la Ría de Huelva en el contexto de las relaciones de intercambio y de las transformaciones producidas en la transición Bronce Final/Edad del Hierro", en M. RuizGálvez (ed.): Ritos de Paso y Puntos de Paso. La Ría de Huelva en el mundo del Bronce Final Europeo. Complutum, Extra 5. Madrid: 129-155.

Ruiz-Gálvez, Mª (2013): Con el fenicio en los talones. Los inicios de la Edad del Hierro en la cuenca del Mediterráneo. Edicions Bellaterra, Barcelona.

Ruiz Zapatero, G. (1992a): “Comercio protohistórico e 
innovación tecnológica: la difusión de la metalurgia del hierro y el torno de alfarero en el NE. de Iberia". Gala. Revista d'Arquelogía i Antropologia, 1: 103-116.

Ruiz Zapatero, G. (1992b): “El Bronce Final y la Primera Edad del Hierro del Valle del Ebro: aprendiendo treinta años después". Homenatge al Prof. Dr. Joan Maluquer de Motes. Pyrenae, 23: 93-97.

Ruiz Zapatero, G. (2007): “Antes del Hierro. Cultura y sociedad en el centro de la Meseta (ca. 1200-500 a.C.)", en A. Dávila (ed.): Estudios sobre la Edad del Hierro en la Carpetania. Museo Arqueológico Regional, Alcalá de Henares: Zona Arqueológica 10, I: 36-62.

Ruiz Zapatero, G.; Fernández Götz, M. y Álvarez-Sanchís, J. R. (2012): "Die Ausbreitung der Eisenmetallurgie auf der Iberischen Halbinsel”, en A. Kern, J.K. Koch, I. Balzer, J. Fries-Knoblach, K. Kovarik, Ch. Later, P.C. Ramsl, P. Trebsche and J. Wiethold (eds.): Technologieentwicklung und-transfer in der Hallstatt-Latènezeit. Beiträge zur Ur- und Frühgeschichte Mitteleuropas 65, Beier und Beran, Langenweissbach: 149-166.

Ruiz Zapatero, G. y Rovira, S. (1996): “La producción, la circulación y el control del metal: del Bronce Medio a la Edad del Hierro en el NE. de la Península Ibérica”. Gala, 3 (5): 33-48.

Sardá, S. (2010): Pràctiques de consum ritual al curs inferior de l'Ebre (s. VII-VI anE). Comensalitat, ideologia i canvi social, Tesis Doctoral, Universitat Rovirai Virgili, Tarragona.

Schattner, T. (2011): " $\mathrm{Zu}$ den Kesselwagen von Baiôes". Madrider Mitteilungen, 52: 266-305.

Schubart, H. (1985): "El asentamiento fenicio del siglo VIII a.C. en el Morro de Mezquitilla (Algarrobo, Málaga)". Aula Orientalis, 3: 63 ss.

Silva, A.C.F.; Silva, C.T.; Lopes, A.B. (1984): "Depósito de fundidor do final da Idade do Bronze do castro da Senhora da Guia (Baiôes, S. Pedro do Sul, Viseu)". Lucerna - Homenagem a D. Domingos de Pinho Brandâo: 73-109.

Snodgrass, A. M. (1980): "Iron and early metallurgy in the Mediterranean", en Th Wertime y J.D. Mulhy (eds.): The Coming of the Age of Iron. Yale University Press. New Haven: 335-374.

Soler, J.Ma (1965): El tesoro de Villena. Excavaciones Arqueológicas en España, 36. Dirección General de Bellas Artes, Ministerio de Educación Nacional. Madrid.

Toledo, A. y Palol, P. de (2006): La necrópolis d'incineració del Bronze Final transició a l'edat del Ferro de Can Bech de Baix, Agullana (Alt Empordá, Girona). Els resultats de la campanya d'excavació de 1974. Museu d'Arqueologia de Catalunya. Sèrie Monogràfica, 24. Girona.

Torres, J. de (2013): La Tierra sin Límites. Territorio, Sociedad e Identidades en el Valle Medio del Tajo (s. IX-I a.C.). Zona Arqueológica, 16. Museo
Arqueológico Regional de la Comunidad de Madrid. Alcalá de Henares.

Torres, M. (2002): Tartessos. Bibliotheca Archaeologica Hispana, 14. Real Academia de la Historia, Madrid.

Torres, M. (2008): "Urnas de tipo Cruz del Negro", en M. Almagro-Gorbea (dir.): La necrópolis de Medellín. II. Estudio de los hallazgos, Bibliotheca Archaeologica Hispana 26-2, Real Academia de la Historia, Madrid: 631-654.

Valle, A. y Serna, M.L. (2003): "El castro de Castilnegro y otros asentamientos de la Edad del Hierro en el entorno de la Bahía de Santander”, en C. Fernández Ibáñez y J. Ruiz Cobo (eds.): La arqueología de la Bahía de Santander. T.I., Santander: 353-390.

Van der Leeuw, S. (2010): "The archaeology of innovation. Lessons for our times", en Moss, F. y Machover, T. (eds.): Innovation: perspectives for the $21^{\text {st }}$ Century. Madrid, BBVA: 33-53.

Vilaça, R. (2006): "Artefactos de ferro em contextos do Bronze Final do território português: Novos contributos e reavaliaçao dos dados". Complutum, 17: 81-101.

Vilaça, R. (2012): “Late Bronze Age: Mediterranean impact in the Western End of the Iberian Peninsula (Actions and Reactions)", en M. E. Aubet y P. Sureda (eds.): Interacciòn social y comercio en la antesala del colonialismo [Actas seminario internacional celebrado en la universidad Pompeu fabra el 28 y 29 de marzo del 2012]. Quadernos de Arqueologia mediterranea, 21: 13-42.

Waldbaum, J.C. (1980): "The first archaeological appearance of iron", en Th Wertime y J.D. Mulhy (eds.): The Coming of the Age of Iron. Yale University Press. New Haven: 69-89.

Waldbaum, J. C. (1999): "The coming of iron in the Eastern Mediterranean: thirty years of archaeological and technological research", en V. C. Piggott (ed.): The Archaeometallurgy of the Asian World. Masca Research Papers in Science and Archaeology, vol 16. The University Museum, University of Pennsylvania, Philadelphia: 27-57.

Wells, P.S. (1980): Culture Contact and Culture Change. Early Iron Age Central Europe and the Mediterranean World. Cambridge University Press.

Wertime, Th. A. y Muhly, J. D. Eds. (1980): The Coming of the age of iron. Yale University Press.

Yalçin, Ü. (1998): "Frühe Eisenverwendung in Anatolien”. Istanbuler Mitteilungen, 48: 79-95.

Zvelebil, M. y Rowley-Conwy, P. (1986): "Foragers and Farmers in Atlantic Europe", en M. Zvelebil (ed.): Hunters in Transition. Cambridge University Press. Cambridge: 67-93. 